\title{
Estudio holístico de la producción de papel a partir de cáñamo industrial en el contexto colombiano
}

\author{
Holistic Study on the Production of Paper from Industrial Hemp in Colombia
}

\author{
Johana Catalina Manosalva Barrera ${ }^{\mathrm{ab}}$, Javier Andrés Dávila ${ }^{\mathrm{ac}}$ \\ y Julián Andrés Quintero ${ }^{d}$
}

\footnotetext{
a Grupo de investigación en Ingeniería de Procesos y Sistemas Industriales, Maestría en Ingeniería de Procesos y Sistemas Industriales, Departamento de Ingeniería, Facultad de Ciencias Naturales e Ingeniería, Universidad de Bogotá Jorge Tadeo Lozano, Colombia

bjohanac.manosalvab@utadeo.edu.co https://orcid.org/0000-0002-0012-0112

cjaviera.davilar@utadeo.edu.co https://orcid.org/0000-0002-4583-7512

d Departamento de Ingeniería Química, Universidad de Santiago de Chile, Chile julian.quintero@usach.cl https://orcid.org/00000003-1224-5649
}

\section{RESUMEN}

El desarrollo y la investigación de nuevos productos que puedan sustituir parcial o completamente materias primas empleadas para la producción de energía, productos químicos de alto valor añadido e incluso la fabricación de papel, ha permitido que el cáñamo industrial (Cannabis sativa L.) se vuelva atractivo como principal materia prima en la reactivación de una economía de base biológica con altas condiciones y capacidades de producción. A partir de lo anterior, el objetivo de este trabajo investigativo es profundizar sobre el potencial del uso de fibra de cáñamo en la industria del papel, soportados en estudios realizados por el Estado Colombiano en lo referente a las disponibilidad de cultivos forestales con fines comerciales, que inicialmente se destinan para la siembra y producción de recursos madereros como el pino y eucalipto. Se ha considerado de importancia demostrar que el cáñamo, como fuente de producción primaria, brinda ventajas competitivas económicas, ambientales y so-

Citation: Manosalva-Barrera, J. C., Dávila, J. A. y Quintero, J. A. (2020). Estudio holístico de la producción de papel a partir de cáñamo industrial en el contexto colombiano. Mutis, 10(2), 51-69. https://doi. org/10.21789/22561498.1721

Recibido: Diciembre 7, 2020. Aceptado: Diciembre 30, 2020.

Copyright: @2020. Manosalva-Barrera, J. C., Dávila, J. A. y Quintero, J. A. (2020). This is an open-access article, which permits unrestricted use, distributions and reproduction in any medium, provided the original author and source are credited.

Competing Interests: The authors have no conflict of interest. ciales frente a los cultivos tradicionales, incluso para las nuevas fuentes de investigación de base biológica.

Palabras claves: cáñamo, celulosa, papel, economía verde, holístico.

\section{ABSTRACT}

The research and development of new products that could partially or completely replace raw materials used for energy production, high 
value-added chemical products, and even the production of paper, has allowed industrial hemp (Cannabis sativa L.) to become the main raw material for the reactivation of a bio-based economy with high production conditions and capacities. Based on this, the objective of this research is to deepen on the potential use of hemp fiber in the paper industry, supported by studies carried out by the Colombian State regarding the availability of forest crops for commercial purposes, which are basically used for planting and the production of wood resources such as pine and eucalyptus. It has been considered important to demonstrate that hemp, as a source of primary production, provides competitive economic, environmental, and social advantages over traditional crops, even for new sources of bio-based research.

Keywords: Hemp, cellulose, paper, green economy, holistic.

\section{INTRODUCCIÓN}

En los últimos años, el sector papelero se ha venido enfrentando a varios problemas relacionados con la escasez de recursos forestales tradicionales, principalmente la fibra de celulosa para la producción de papel. Por ello, el impacto ambiental de esta industria es una de las causas fundamentales de la problemática a tratar (González-García et al., 2010a). Aunque la madera sigue siendo la materia prima esencial en la producción de papel, el consumo y las investigaciones en torno a fibras no madereras han venido mostrando una tendencia creciente en los últimos años (Plazonić et al., 2016). Los problemas ambientales generados por el uso a gran escala de madera han llevado a la industria del papel a enfocarse estratégicamente en el camino del desarrollo sostenible, aunando esfuerzos para reducir significativamente su impacto ambiental y la escases ecológica mediante el uso de especies de fibra de alta calidad y crecimiento más rápido (que los recursos tradicionales) como materias primas alternativas.

El Cannabis sativa L., conocido como cáñamo industrial, es un material orgánico que presenta concentraciones significativas de polisacáridos (lignina, hemicelulosa y celulosa), los cuales son componentes básicos de sus fibras naturales. Esta especie además contiene extractos y otros compuestos que pueden ser el punto de partida para el desarrollo de productos con alto valor agregado (Kitrytè et al., 2018). Adicionalmente, su cepa contiene un bajo nivel (hasta 0,3\%) del principal componente psicoactivo de este tipo de plantas, el tetrahidrocannabinol (THC), lo cual hace idóneo su uso para fines industriales. Prueba de ello es que el mercado global de cáñamo consta de más de 25.000 productos en nueve submercados: agricultura, textiles, reciclaje, automotriz, muebles, alimentos y bebi- das, papel, materiales de construcción y cuidado personal (Johnson, 2018).

El cáñamo es un recurso no maderero con factores característicos desde la siembra, tales como un consumo mínimo de agua, un rápido crecimiento de la planta (en comparación con las plantaciones de eucalipto, pino y caña de azúcar, las cuales son actualmente la materia base de la industria papelera) (Becerra-Quiroz et al., 2016), versatilidad, fácil manipulación y un rendimiento superior al de otros cultivos no madereros.

En Colombia se han proyectado inversiones de millones de dólares por parte de empresas multinacionales, las cuales ven en el país un punto focal para la producción, comercialización y exportación de productos a base de cannabis no psicoactivo. Ventajas como sus condiciones climáticas y geográficas y el aumento de acuerdos comerciales y legislativos, convierten al país en un lugar estratégico para el crecimiento de la producción de cáñamo.

Expuesto lo anterior, el objetivo de este trabajo es evaluar el uso del cáñamo como fuente primaria en la obtención de pulpa para la producción de papel y su pertinencia como sustituto o complemento de las materias primas convencionales empleadas en la industria colombiana del papel. Para ello, se caracteriza el material lignocelulósico con respecto al contenido de celulosa, hemicelulosa, lignina, humedad, extractivos y ceniza (Moonart \& Utara, 2019), obteniendo papel en laboratorio a partir de la fibra de cáñamo. Lo anterior fue posible gracias a la aplicación de metodologías convencionales, empleando el método Kraft para la obtención de pulpa y realizando una evaluación holística del sistema agrícola del Cannabis sativa $\mathrm{L}$. 


\section{MATERIALES Y MÉTODOS}

\section{Caracterización del material lignocelulósico}

Se plantea la metodología propuesta por el National Renewable Energy Laboratory (NREL), que indicará el contenido de componentes aprovechables, tal como celulosa, hemicelulosa, humedad, extractivos y cenizas.

Antes de cada procedimiento, el cáñamo fue sometido a un tratamiento de secado a $80{ }^{\circ} \mathrm{C}$ durante 48 horas, procediendo luego a la molienda. El contenido de humedad se determinó mediante el uso de un horno convencional en el que se sometió el cáñamo a una temperatura de $105{ }^{\circ} \mathrm{C}$ durante 24 horas. Las muestras se conservaron en un desecador durante 1 hora para alcanzar la temperatura ambiente. Posteriormente, estas fueron pesadas y se procedió a calcular su contenido de humedad siguiendo el protocolo establecido por la NREL (Partisi, 2008).

Los extractos acuosos y de etanol se determinaron utilizando una extracción Soxhlet con agua y etanol, respectivamente, durante 24 horas por triplicado (Han \& Rowell, 2008). Los extractos de etanol contienen clorofila y pigmentos naturales (Dávila et al., 2017), por lo que el contenido total es la suma de los extractivos solubles en agua y etanol.

El contenido de lignina se determinó a partir de una deslignificación con ácido sulfúrico $\left(\mathrm{H}_{2} \mathrm{SO}_{4}\right)$ al $72 \%$ por 1 hora. La muestra se trata en un autoclave y se filtra. Posterior a esto, los residuos se lavan con agua caliente y se secan a $105^{\circ} \mathrm{C}$ (Han \& Rowell, 2008).

El contenido de holocelulosa se determinó con el uso de clorito de sodio $\left(\mathrm{NaClO}_{2}\right)$ al $80 \%$ y ácido acético al $96 \%$; ambos reactivos se agregaron a la muestra cada hora durante 7 horas. Seguido, la mezcla se filtra y se seca a $105^{\circ} \mathrm{C}$.

El contenido de celulosa se obtuvo agregando hidróxido de sodio $(\mathrm{NaOH})$ al $17,5 \%$ cada 5 minutos por 1 hora, a $20^{\circ} \mathrm{C}$. Luego se filtra y se seca a $40{ }^{\circ} \mathrm{C}$. La diferencia entre el contenido de holocelulosa y celulosa es el contenido de hemicelulosa en la muestra. Finalmente, el contenido de ceniza se obtuvo después de incinerar la muestra a $575^{\circ} \mathrm{C}$ durante 3 horas, tras lo cual esta se pasa a un desecador por 30 minutos y es pesada. El proceso se realizó por triplicado (Dávila et al., 2017).

\section{Obtención de pasta de celulosa para la fabricación de papel}

La obtención de pulpa de celulosa para la producción de papel involucra el desarrollo de pruebas preliminares con material lignocelulósico. El objetivo de dichas pruebas es conocer las variables que afectan la obtención de la pulpa, adquirir experiencia para mejorar en el proceso productivo del papel a base de cáñamo y estandarizar el proceso de obtención de celulosa para fines productivos.

La producción de papel se realizará a través del método Kraft, el cual permitirá obtener papel con una máxima eficiencia del material lignocelulósico en comparación con otros métodos industriales empleados para la producción de pasta de celulosa, como el tipo sulfito o el método de soda (Chávez-Sifontes \& Domine, 2010). El método Kraft consiste en:

- Acondicionamiento de la materia prima (determinar el contenido de humedad), donde esta es previamente tratada para la disminución del tamaño de partícula.

- Preparación del licor blanco para la digestión, que involucra alimentación de la materia prima, acondicionamiento del reactor $\left(\mathrm{P}=40 \mathrm{psi} ; \mathrm{T}=140{ }^{\circ} \mathrm{F}\right)$ y un tiempo de reacción de 4 horas (van-der-Werf et al., 1994).

- Proceso de cocción.

- Suspensión del proceso, permitiendo que la presión y la temperatura desciendan antes de abrir el reactor.

- Separación de la parte sólida y líquida (separación de la pulpa y el licor negro).

- Blanqueamiento de la pulpa, donde esta es lavada y blanqueada tres veces con hipoclorito de sodio al $13 \%$.

- Homogenización y laminado de la pulpa, donde se homogeniza y se filtra la pulpa, para posteriormente laminarla de forma artesanal.

- Obtención del producto final. 
La primera prueba preliminar realizada (Caso 1) se determinó con un peso del $40 \%$ para el hidróxido de sodio, $8 \%$ para el sulfuro de sodio y $12 \%$ para el carbonato de calcio. Determinando las cantidades de los reactivos necesarios para una masa de $60 \mathrm{~g}$ de aserrín de pino a un litro de licor, se realiza el respectivo proceso de reacción. Inicialmente, se toman los valores del Caso 1, se determina la humedad, el número de lavados a realizar y el tipo de reactivo para el proceso de blanqueado (tabla 1).

Tabla 1. Condiciones iniciales de la reacción Caso 1 - Relación 3:1

\begin{tabular}{|c|c|}
\hline Peso de la muestra inicial (g) & 60 \\
\hline $\mathrm{NaOH}(\mathrm{g})$ & 120 \\
\hline $\mathrm{Na} 2 \mathrm{~S}(\mathrm{~g})$ & 24 \\
\hline $\mathrm{CaCO}_{3}(\mathrm{~g})$ & 36 \\
\hline $\mathrm{N}^{\circ}{ }^{\circ}$ lavados & 3 \\
\hline Reactivo & Hipoclorito de sodio $(13 \%)$ \\
\hline
\end{tabular}

Fuente: elaboración propia.

\section{Estudio holístico del proceso agrícola del cáñamo}

\section{Zonificación de las plantaciones forestales comerciales}

La delimitación de las hectáreas y zonas destinadas para los cultivos de plantaciones forestales con usos comerciales se basó en un estudio realizado por la Unidad de Planificación Rural Agropecuaria (UPRA), titulado "Lineamientos de política: Plantaciones forestales con fines comerciales para la obtención de madera y su cadena productiva", en el cual se ha optado por clasificar las plantaciones forestales en tres categorías de acuerdo con el nivel de aptitud de los terrenos: A1 (aptitud alta), A2 (aptitud media) y A3 (aptitud baja) (UPRA, 2018).

\section{Simulación del sistema agrícola del cáñamo}

Con el fin de analizar los impactos directos del sistema agrícola del cáñamo, así como optimizar e identificar mejoras en caso de que la cadena de producción en las zonas destinadas para tal fin se llevara a cabo con este cultivo (Díaz-Martínez \& Zárate-Cruz, 2018), la simulación se desarrollará empleando el software FlexSim, en donde se contemplan los procesos de los dos subsistemas relevantes: producción agrícola y tratamiento del material.

\section{Toma de decisiones}

Para desarrollar un criterio que permita tomar la decisión de potencializar el cultivo de cáñamo como material, en aras de generar una economía verde a gran escala y reemplazar los cultivos de plantaciones tradicionales, se utilizará una matriz multicriterio desarrollada en el software Expert Choice (Tavella et al., 2016) y la metodología de procesos jerárquicos, también conocida como Analytic Hierarchy Process (AHP).

\section{RESULTADOS Y DISCUSIÓN}

Caracterización del material lignocelulósico con respecto al contenido de celulosa, hemicelulosa, lignina, humedad, extractivos y ceniza

El cáñamo, como materia prima, fue producido en un autocultivo ubicado en Silvania (Cundinamarca, Colombia). La matriz vegetal fue fresada utilizando un molino de impacto SK 100 (RETSCH, Alemania) y tamizada por una serie de tamices Tyler (ASTM E-11) de malla 20-80 $\mu \mathrm{m}$. Luego, la muestra se conservó a $-4{ }^{\circ} \mathrm{C}$ (figura 1).

De acuerdo con la metodología planteada y descrita por la NREL, en la tabla 2 se detallan los resultados obtenidos en lo referente a la caracterización del cáñamo.

Tabla 2. Caracterización química del cáñamo

\begin{tabular}{|c|c|c|}
\hline Componente & Contenido (\%) & Desviación estándar \\
\hline Lignina & 12,8 & $\pm 0,0050$ \\
\hline Celulosa & 50,3 & $\pm 0,0043$ \\
\hline Hemicelulosa & 27,8 & $\pm 0,0032$ \\
\hline Cenizas & 2,3 & $\pm 0,0020$ \\
\hline Extractivos & 6,8 & $\pm 0,0420$ \\
\hline Total & 100 & \\
\hline
\end{tabular}

Fuente: elaboración propia. 
Tallos secos de cáñamo

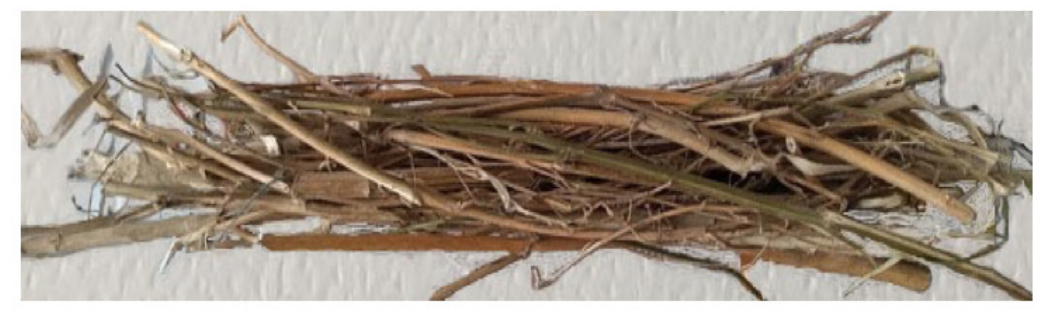

Cáñamo molido

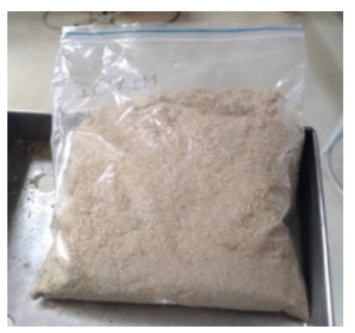

Figura 1. Tallos y cáñamo molido Fuente: elaboración propia.

\section{Lignina}

El contenido de lignina fue de $12,8 \%$. Comparado con registros de materiales lignocelulósicos similares, como la madera (15-25\%), el bagazo de caña (14 \%) y la paja de arroz (6,1 \%) (Chávez-Sifontes \& Domine, 2010), este contenido es un indicativo de que el cáñamo representa un recurso renovable con potencial de uso industrial, puesto que la lignina tiene aplicaciones que van desde la producción de etanol a partir de la biomasa lignocelulósica, la producción de dispersantes de pesticidas, emulsificantes y secuestradores de metales pesados y la composición de formulaciones para mejorar las propiedades de desempeño de resinas o adhesivos, hasta la producción de carbón activado (Chávez-Sifontes \& Domine, 2010).

\section{Celulosa}

Los resultados obtenidos indican que el cáñamo es una excelente fuente de celulosa (50,3\%) en comparación con otros materiales lignocelulósicos, como el bambú (34,5\%), el coco (46\%) (Komuraiah et al., 2014), la paja de trigo (30\%), las hojas de maíz (45\%), el residuo de la caña de azúcar (40 \%) (Daza-Merchán et al., 2013) e incluso la madera (38-50 \%) (Chávez-Sifontes \& Domine, 2010), los cuales son las materias primas de mayor potencial en la industria. Es de importancia destacar que no todos los residuos orgánicos son favorables para la obtención de pulpa para la producción de papel. Así, los residuos con potencial de producción están por encima del $44 \%$ de celulosa (González-Velandia et al., 2016).

\section{Hemicelulosa}

El cáñamo registra un contenido de hemicelulosa de $27,8 \%$, un porcentaje superior al registrado por algu- nos materiales lignocelulósicos con potencial en la industria, como las maderas blandas (23-31\%), las maderas duras (20-40\%), la paja (23-38\%), la hoja de tusa del maíz (11-31 \%), la corona de piña (14-50 \%) y el tallo de maíz (20 \%) (Cebreiros et al., 2020). Dicho porcentaje de hemicelulosa convierte al cáñamo en material adecuado para la producción de biocombustibles y otros productos derivados del azúcar, tales como bioetanol, biobutanol, furfural y xilital, entre otros (Cebreiros et al., 2020), o como materia prima para la producción de azúcares reductores (Dávila et al., 2017).

\section{Cenizas}

Según algunos estudios, el contenido de cenizas de toda la planta debe ser de hasta $3 \%$, y este no debe ser superior a 5-7 \% para que pueda utilizarse como fertilizante $u$ otro subproducto (Sausserde \& Adamovics, 2013). En este estudio, el contenido de cenizas del cáñamo (2,3\%) está dentro de los parámetros. Además, el fertilizante obtenido de su ceniza puede ser muy valioso, ya que contiene $24 \%$ de óxido de calcio ( $\mathrm{CaO}), 4,85 \%$ de óxido fosfórico $\left(\mathrm{P}_{2} \mathrm{O}_{5}\right)$ y $6,3 \%$ de óxido de potasio ( $\left.\mathrm{K}_{2} \mathrm{O}\right)$ (Poisa \& Adamovics, 2011).

\section{Obtención de papel a partir de aserrín de pino y cáñamo}

\section{Obtención de papel a partir de pino}

La reacción llevó un tiempo de 4 horas a una presión de 40 psi y una temperatura de $140{ }^{\circ} \mathrm{F}$, más 30 minutos en espera de la condensación de vapores y el enfriamiento del equipo. Tras obtener el residuo de la reacción, se realiza el primer lavado con agua a fin de eliminar los residuos de ácido que este obtuvo en el proceso de cocción. 
Luego, se alternan los blanqueados con hipoclorito de sodio a una concentración de $13 \%$ y con agua, nuevamente, hasta completar 3 ciclos de lavados.
En lo concerniente a este estudio, los casos (1, 2 y 3 ) son los pesos de los reactivos a tener en cuenta para la preparación del licor de cocción, como se observa en la tabla 3.

Tabla 3. Caracterización química del cáñamo

\begin{tabular}{|c|c|c|c|c|}
\hline Reactivos & Rango porcentaje recomendado & Caso 1 & Caso 2 & Caso 3 \\
\hline $\mathrm{NaOH}$ & $40-60 \%$ & 0,40 & 0,50 & 0,60 \\
\hline $\mathrm{Na}_{2} \mathrm{~S}$ & $8-15 \%$ & 0,08 & 0,12 & 0,15 \\
\hline $\mathrm{CaCO}_{3}$ & $12-20 \%$ & 0,12 & 0,16 & 0,20 \\
\hline Total & & $\mathbf{0 , 6 0}$ & $\mathbf{0 , 7 8}$ & $\mathbf{0 , 9 5}$ \\
\hline
\end{tabular}

Fuente: elaboración propia.

\section{Primer ensayo. Relación 3:1 con aserrín de pino, Caso 1}

El primer ensayo se realizó con $60 \mathrm{~g}$ de pino a una relación másica 3:1, obteniendo como resultado la composición del licor de cocción así: hidróxido de sodio $(\mathrm{NaOH})$ de $40 \%=120 \mathrm{~g}$; sulfuro de sodio $\left(\mathrm{Na}_{2} \mathrm{~S}\right)$ de $8 \%=24$ g; carbonato de calcio $\left(\mathrm{CaCO}_{3}\right)$ de $12 \%=36$ g. En la figura 2 se observa el cambio de color que se presentó en cada etapa de blanqueado. Para finalizar el proceso, se prosiguió a laminar de forma artesanal la pasta obtenida en un molde de aluminio, donde la pasta se conservó por 12 horas en la prensa. La lámina obtenida se muestra en la figura 3.

El producto final registró un peso de 15,56 g. De lo anterior, se puede establecer que el rendimiento del proceso fue de aproximadamente $25,88 \%$. Debido al bajo rendimiento obtenido, se propone que en el siguiente ensayo se aumente la relación másica entre la materia prima y el licor blanco a 5:1, considerando que la reacción de cocción no fue eficiente frente a la cantidad de masa inicial.
Primer lavado con hipoclorito

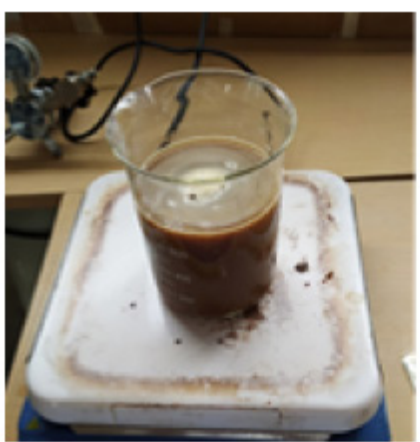

Segundo lavado con hipoclorito

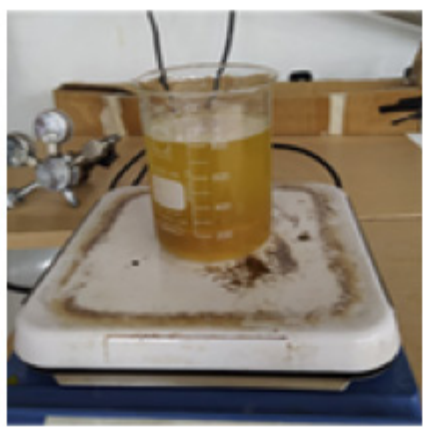

Tercer lavado con hipoclorito

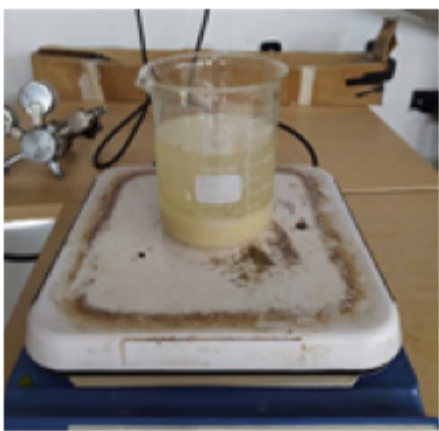

Figura 2. Proceso de blanqueado del primer ensayo preliminar con pino Fuente: elaboración propia.

\section{Segundo ensayo. Relación 5:1 con aserrín de pino, Caso 1}

La segunda prueba preliminar con aserrín de pino se desarrolló con una relación másica de 5:1 del licor de cocción y la materia prima. Se determinó la cantidad de reactivos necesarios para una masa de $60 \mathrm{~g}$ de aserrín de pino a un litro de licor. Además, se realizó el respectivo proceso de reacción descrito en la metodología del objetivo. Teniendo en cuenta lo anterior, los parámetros iniciales del proceso para el segundo ensayo se describen en la tabla 4. Por su parte, en la figura 4 se observa el cambio de color que se presentó en cada blanqueado. 


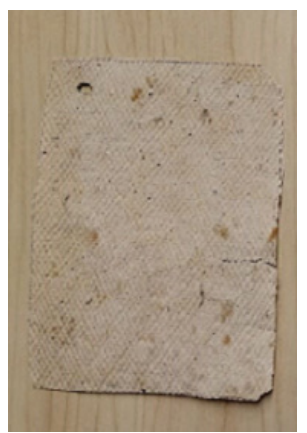

Figura 3. Lámina de pasta de celulosa del segundo ensayo preliminar con pino

Fuente: elaboración propia.
Tabla 4. Condiciones iniciales de la reacción Caso 1 - Relación 5:1

\begin{tabular}{|c|c|}
\hline Peso de la muestra inicial $(\mathrm{g})$ & 60 \\
\hline $\mathrm{NaOH}(\mathrm{g})$ & 200 \\
\hline $\mathrm{Na}_{2} \mathrm{~S}(\mathrm{~g})$ & 40 \\
\hline $\mathrm{CaCO}_{3}(\mathrm{~g})$ & 60 \\
\hline $\mathrm{No}$. lavados & 3 \\
\hline Reactivo & Hipoclorito de sodio $(13 \%)$ \\
\hline
\end{tabular}

Fuente: elaboración propia.

De este ensayo no se obtuvo un resultado final. Se presenció cuando se realizaban los lavados que la cantidad de material disminuía, por lo cual, al llegar al tercer lavado con hipoclorito, no se obtuvo material suficiente para obtener la lámina. Dado lo anterior, nos limitamos a realizar hacer ensayos con la relación másica 5:1.
Primer lavado con hipoclorito

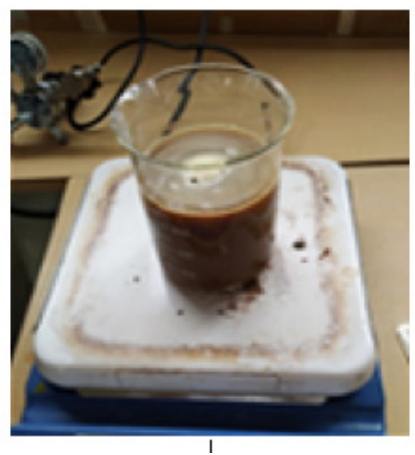

Segundo lavado con hipoclorito

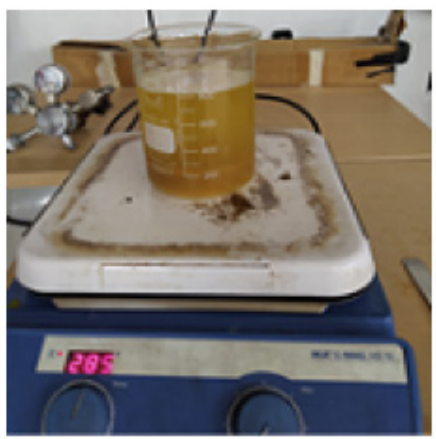

Tercer lavado con hipoclorito

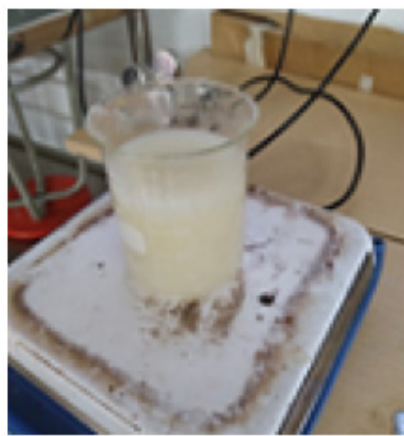

Figura 4. Proceso de blanqueado del segundo ensayo preliminar con pino Fuente: elaboración propia.

\section{Tercer ensayo. Relación 3:1 con aserrín de pino, Caso 2}

Para las pruebas preliminares del tercer ensayo se disminuyó la cantidad de material inicial a $30 \mathrm{~g}$ de aserrín de pino para un volumen de un litro, con una relación 3:1. Para este ensayo se emplearon los valores intermedios de los porcentajes de los reactivos, obteniendo las cantidades que se observan en la tabla 5 . El proceso obtenido se observa en la figura 5.

Tabla 5. Condiciones iniciales del tercer ensayo, Caso 2 - Relación 3:1

\begin{tabular}{|c|c|c|c|c|c|c|c|}
\hline Reactivos & Rango porcentaje recomendado & Caso 1 & Caso 2 & Caso 3 & Caso 1 & Caso 2 & Caso 3 \\
\hline $\mathrm{NaOH}$ & $40-60 \%$ & 0,40 & 0,50 & 0,60 & 60 & 58,1 & 56,8 \\
\hline $\mathrm{Na} 2 \mathrm{~S}$ & $8-15 \%$ & 0,08 & 0,12 & 0,15 & 12 & 13,4 & 14,2 \\
\hline $\mathrm{CaCO}_{3}$ & $12-20 \%$ & 0,12 & 0,16 & 0,20 & 18 & 18,6 & 18,9 \\
\hline & Total & $\mathbf{0 , 6 0}$ & $\mathbf{0 , 7 8}$ & $\mathbf{0 , 9 5}$ & $\mathbf{9 0}$ & $\mathbf{9 0}$ & $\mathbf{9 0}$ \\
\hline
\end{tabular}

Fuente: elaboración propia. 
Primer lavado con hipoclorito

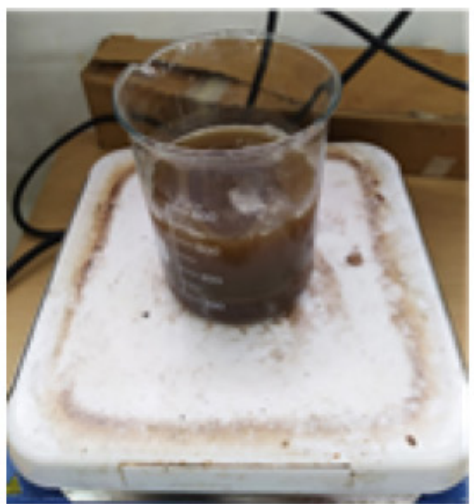

Segundo lavado con hipoclorito

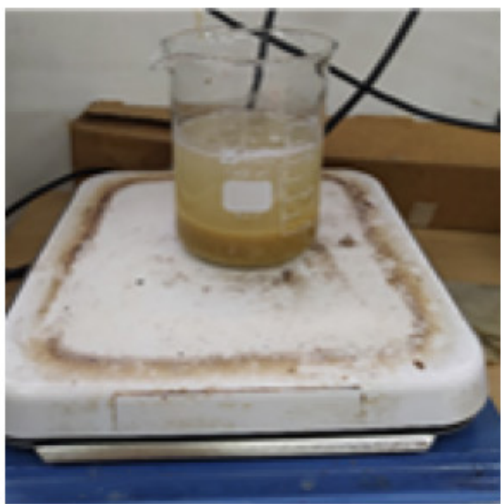

Tercer lavado con hipoclorito

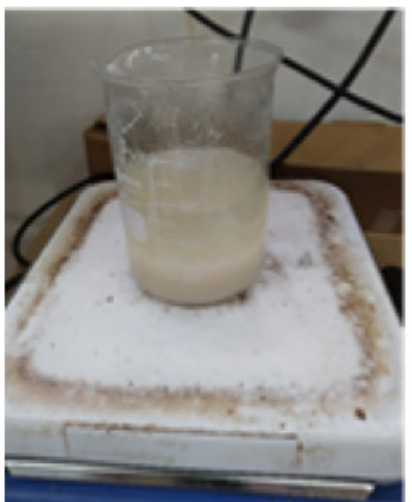

Figura 5. Proceso de blanqueado del tercer ensayo preliminar con pino Fuente: elaboración propia.

Se obtuvo una lámina (figura 6) tras aplicar los procedimientos establecidos. El producto final obtuvo un peso de $11 \mathrm{~g}$. De lo anterior, se puede establecer que el rendimiento del proceso fue de aproximadamente $36,67 \%$.

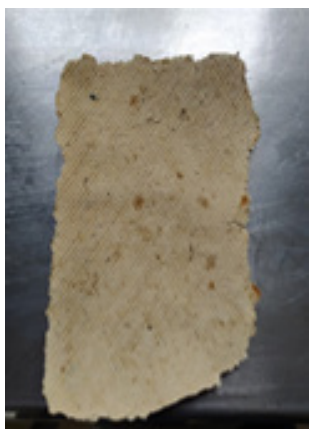

Figura 6. Lámina de pasta de celulosa tras el tercer ensayo preliminar con pino

Fuente: elaboración propia.

\section{Obtención de papel a partir de la fibra de cáñamo}

De acuerdo con los ensayos preliminares realizados con pino, se tomaron consideraciones para el desarrollo de las pruebas con cáñamo, entre las cuales están:

- Debido a que durante el desarrollo de las pruebas preliminares se observó un desperdicio de material en el momento del moldeado a causa de la cantidad de material inicial a trabajar, y de acuerdo con el último ensayo trabajado con $30 \mathrm{~g}$ de materia seca, el cual obtuvo mayor rendimiento, se consideró trabajar las pruebas finales con 30 g de cáñamo.
- Las pruebas se desarrollaron con una relación másica 3:1 debido a que se obtuvo mejor rendimiento con esta proporción.

\section{Primer ensayo. Relación 3:1 con fibra de cáñamo, Caso 1}

La primera prueba final se desarrolló con los parámetros anteriormente expuestos, obteniendo las condiciones del proceso que se detallan en la tabla 6 .

Tabla 6. Condiciones iniciales de la primera prueba final con cáñamo, Caso 1 - Relación 3:1

\begin{tabular}{|c|c|}
\hline Peso de la muestra inicial $(\mathrm{g})$ & 30 \\
\hline $\mathrm{NaOH}(\mathrm{g})$ & 60 \\
\hline $\mathrm{Na} 2 \mathrm{~S}(\mathrm{~g})$ & 12 \\
\hline $\mathrm{CaCO}_{3}(\mathrm{~g})$ & 18 \\
\hline Humedad inicial (\%) & 14,54 \\
\hline $\mathrm{N} .{ }^{\circ}$ lavados & 3 \\
\hline Reactivo & Hipoclorito de sodio (13 \%) \\
\hline
\end{tabular}

Fuente: elaboración propia.

El proceso se llevó a cabo con las mismas condiciones de tiempo $(4 \mathrm{~h})$, temperatura $\left(140^{\circ} \mathrm{F}\right)$ y presión (40 psi). Después de la reacción, se realizaron cuatro lavados con agua y tres blanqueados con hipoclorito de sodio al $13 \%$ para obtener los resultados observados en la figura 8. Para finalizar el proceso, se prosiguió a laminar de forma artesanal la pasta obtenida. Continuando con el proceso, se obtuvo una lámina como la que se muestra en la figura 9. 
Primer lavado con hipoclorito

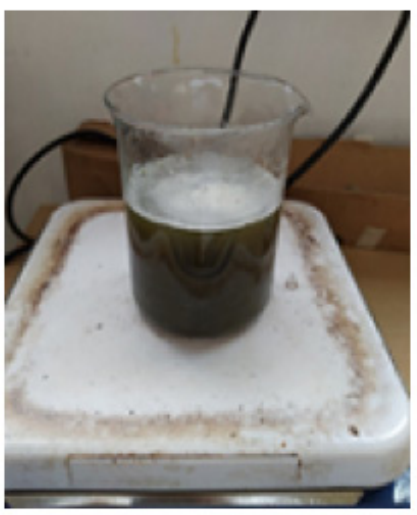

Segundo lavado con hipoclorito

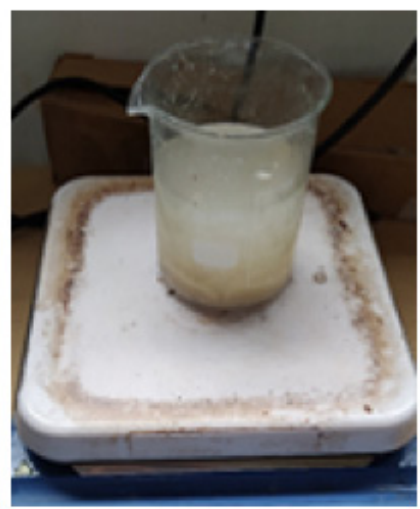

Tercer lavado con hipoclorito

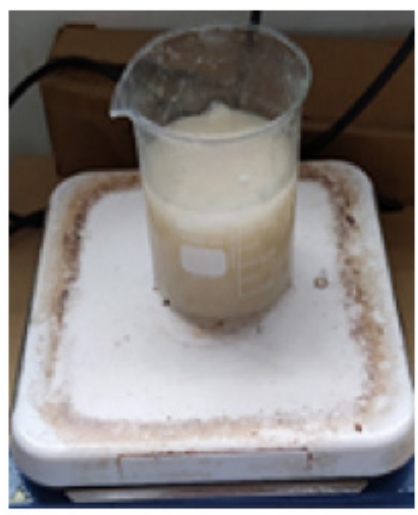

Figura 8. Proceso de blanqueado del primer ensayo con cañamo Fuente: elaboración propia.

Desmoldado

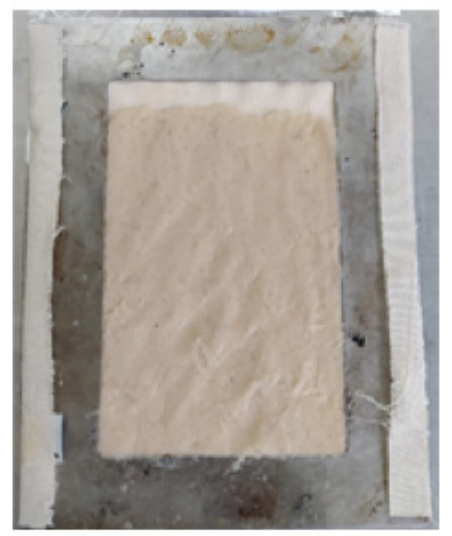

Figura 9. Lámina de pasta primer ensayo con cañamo Fuente: elaboración propia.

Nota: se presentó un inconveniente en el reactor después de 2:30 h de empezar la reacción, debido a fallas en el controlador de presión (fallo). Luego de la reacción de la primera prueba se consideran variables a ajustar, como el número de lavados con hipoclorito de sodio, ya que no se observó ninguna diferencia visual tras realizar dos o tres lavados.

\section{Segundo ensayo. Relación 3:1 con fibra de cáñamo, Caso 1}

Las condiciones del proceso se dan como se detaIla en la tabla 7. La segunda prueba final se desarrolló con los mismos parámetros de tiempo (4h), temperatura $\left(140^{\circ} \mathrm{F}\right.$ ) y presión (40 psi) que en los procesos anteriormente expuestos. Después de la reacción, se realizaron tres lavados con agua y dos blanqueados con hipoclorito de sodio al $13 \%$ para obtener los resultados observados en la figura 10. Continuando con el proceso, se obtuvo una lámina como la que se observa en la figura 11.
Tabla 7. Condiciones iniciales de la segunda prueba con cáñamo, Caso 1 - Relación 3:1

\begin{tabular}{|cc|}
\hline Peso de la muestra inicial (g) & 30,2 \\
\hline $\mathrm{NaOH}(\mathrm{g})$ & 60 \\
\hline $\mathrm{Na} 2 \mathrm{~S}(\mathrm{~g})$ & 12 \\
\hline $\mathrm{CaCO}_{3}(\mathrm{~g})$ & 18 \\
\hline Humedad Inicial (\%) & 14,54 \\
\hline $\mathrm{N} .{ }^{\circ}$ lavados & 3 \\
\hline Reactivo & Hipoclorito de sodio (13\%) \\
\hline
\end{tabular}

Fuente: elaboración propia.

El producto final registró un peso de $14,59 \mathrm{~g}$. A partir de lo anterior, se puede establecer que el rendimiento del proceso fue de aproximadamente $48,6 \%$.

\section{Tercer ensayo. Relación 3:1 con fibra de cáñamo, Caso 1}

La tercera prueba final se desarrolló con los parámetros de tiempo, temperatura y presión señalados en los procesos anteriormente expuestos. Las cantidades de los reactivos y las condiciones a trabajar se observan en la tabla 8. Finalizando el proceso, se obtuvo una lámina como la observada en la figura 12. 
Primer lavado con agua

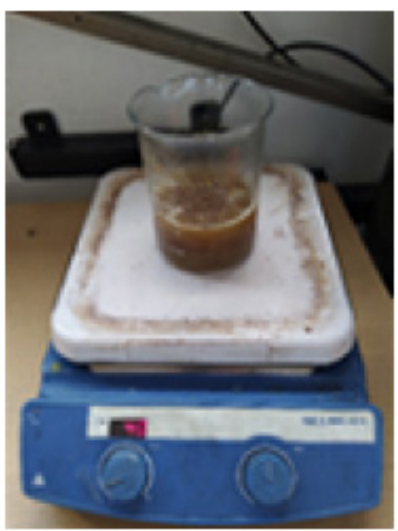

Segundo lavado con hipoclorito

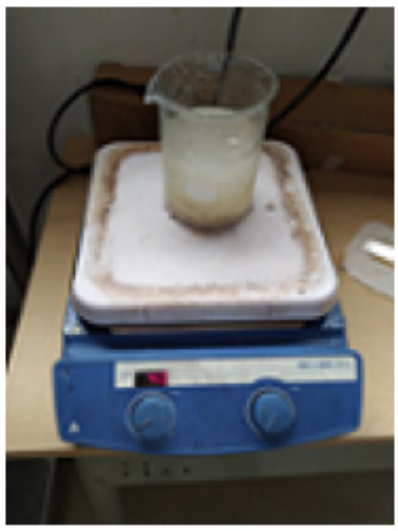

Tercer lavado con hipoclorito

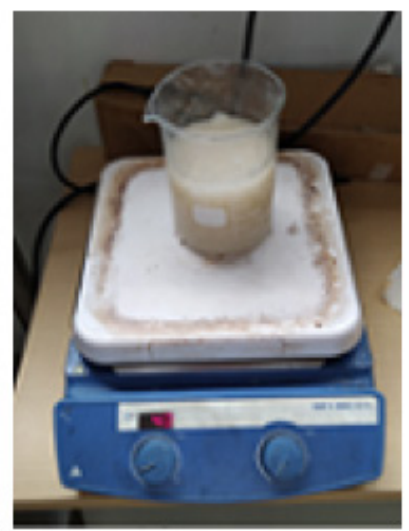

Figura 10. Proceso de blanqueado del segundo ensayo con cáñamo Fuente: elaboración propia.

Desmoldado

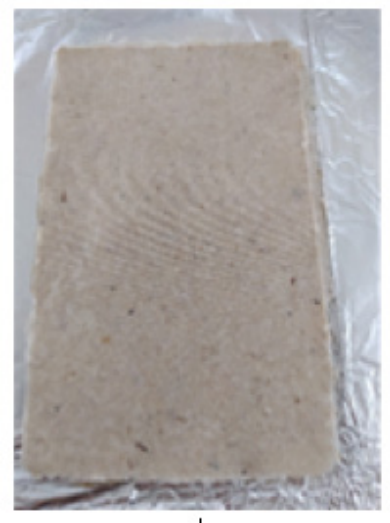

Figura 11. Lámina de pasta del segundo ensayo con cáñamo Fuente: elaboración propia.

Tabla 8. Condiciones iniciales de la tercera prueba con cáñamo, Caso 1 Relación 3:1

\begin{tabular}{|c|c|}
\hline Peso de la muestra inicial $(g)$ & 30 \\
\hline $\mathrm{NaOH}(\mathrm{g})$ & 60 \\
\hline $\mathrm{Na2S}(\mathrm{g})$ & 12 \\
\hline $\mathrm{CaCO}_{3}(\mathrm{~g})$ & 18 \\
\hline Humedad inicial (\%) & 14,54 \\
\hline $\mathrm{N}^{\circ}$. lavados & 3 \\
\hline Reactivo & Hipoclorito de sodio (13 \%) \\
\hline
\end{tabular}

Desmoldado

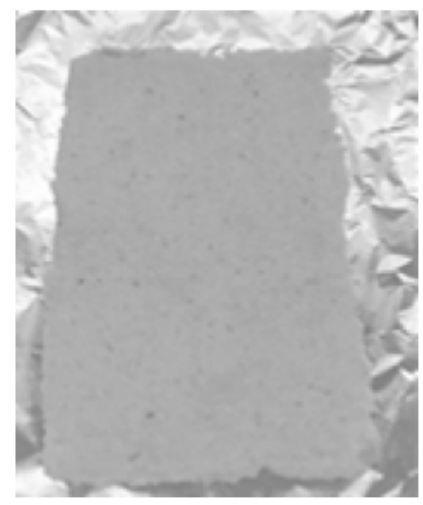

Figura 12. Lámina de pasta del segundo ensayo con cáñamo Fuente: elaboración propia.

Fuente: elaboración propia.

El producto final reportó un peso de $12,9 \mathrm{~g}$. De lo anterior, se puede establecer que el rendimiento del proceso fue de aproximadamente $43 \%$.

\section{Resumen y análisis de los resultados}

De acuerdo con los procesos realizados en los tres ensayos preliminares con pino y las tres pruebas finales con cáñamo, en la tabla 9 se detalla el resumen de los resultados obtenidos, así como las observaciones que se presentaron durante cada proceso. 
Tabla 9. Resumen de resultados obtenidos en todos los ensayos realizados con pino y cáñamo

\begin{tabular}{|c|c|c|c|c|c|c|}
\hline Ensayo & Muestra & Relación & $\begin{array}{c}\text { Peso muestra } \\
\text { inicial (g) }\end{array}$ & $\begin{array}{c}\text { Peso muestra } \\
\text { final (g) }\end{array}$ & $\begin{array}{c}\text { Rendimiento } \\
\text { (\%) }\end{array}$ & Observaciones \\
\hline 1 & Pino & $3: 1$ & 60 & 15,53 & 25,88 & Proceso sin contratiempos \\
\hline 2 & Pino & $5: 1$ & 60 & - & El moldeado no salió completo \\
\hline 3 & Pino & $3: 1$ & 30 & 11 & 36,67 & $\begin{array}{c}\text { Descomposición del material en el tercer lavado con } \\
\text { hipoclorito de sodio }\end{array}$ \\
\hline 1 & Cáñamo & $3: 1$ & 30 & 10,6 & 35,33 & Fallo en el reactor. Dado lo anterior, el material fue \\
insuficiente para cubrir el molde
\end{tabular}

Fuente: elaboración propia.

El cáñamo reporta mayor rendimiento en los procesos de obtención de pulpa de celulosa que el pino bajo las mismas condiciones de tratamiento y con menor cantidad de materia prima inicial. Así, el cáñamo se vuelve atractivo para la industria papelera, si se considera la parte visual del mismo. En la figura 13 se compara una lámina de pino con una de cáñamo, visualizando un color más blanco y uniforme en la muestra de cáñamo.

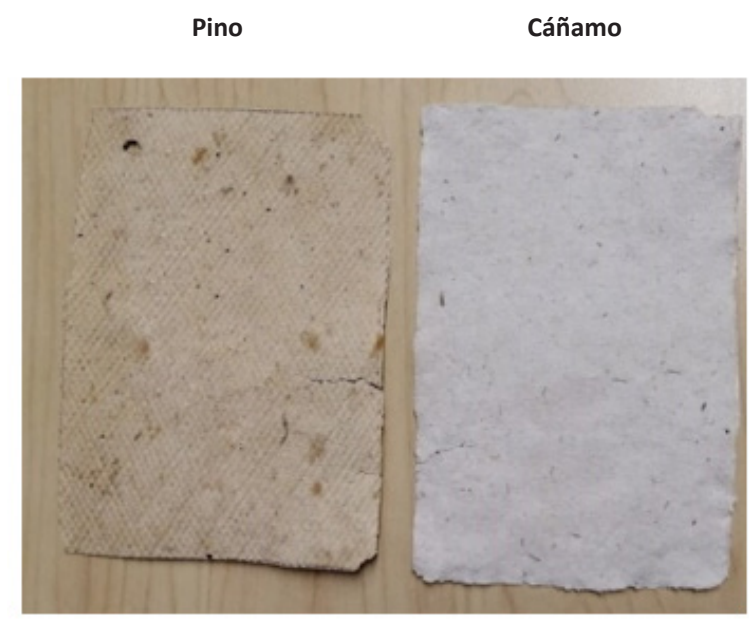

Figura 13. Lámina de pino vs. lámina de cáñamo Fuente: elaboración propia.

De acuerdo con los procesos seguidos para la obtención de pulpa de celulosa a base de pino, este material requirió tres blanqueados con hipoclorito de sodio y cuatro lavados con agua para lograr el color y la viscosidad requeridos. Por su parte, en la segunda prueba con cáñamo se comprobó que el tercer blanqueado con hipoclorito de sodio no era necesario para este material; el color y la viscosidad de la lámina era iguales tras realizar tres blanqueados y cuatro lavados con agua.Lo anterior refleja un ahorro en los procesos de blanqueado de la pulpa, en los reactivos empleados y, por consiguiente, en los costos.

\section{Pruebas gravimétricas}

El gramaje del papel es una característica física ampliamente considerada para contrastar la calidad de los diferentes tipos de papel. El gramaje es el peso del papel por unidad de superficie $\left(\mathrm{g} / \mathrm{m}^{2}\right)$, la cual está determinada por la norma Iso 536: Pesos de papel y gramaje (ISO, 2013). En general, cuanto mayor sea el gramaje del papel, más resistente y gruesa será la hoja. Para la caracterización realizada en esta investigación se contemplaron los parámetros indicados en la tabla 10.

De acuerdo con lo anterior, el papel de cáñamo se clasifica como un "papel con más cuerpo y grosor que el de 80 g., mate o brillante", atributos recomendables para impresiones de alta calidad. Por otra parte, el papel de pino se clasifica como un "papel grueso o cartulina ligera", recomendable para impresiones de certificados, pósteres y cartulinas, entre otros (ISO, 2013). 
Tabla 10. Resumen de resultados obtenidos en los ensayos realizados con pino y cáñamo

\begin{tabular}{|l|c|c|}
\hline & & \\
\hline & & \\
\hline Molde & & \\
\hline Peso muestra $(\mathrm{g})$ & 15.53 & 14.59 \\
\hline Área $\left(\mathrm{m}^{\wedge} 2\right)$ & 0.122 & 0.122 \\
\hline Gramaje $\left(\mathrm{g} / \mathrm{m}^{\wedge} 2\right)$ & 127.3 & 119.6 \\
\hline
\end{tabular}

Fuente: elaboración propia.

\section{Realización de una evaluación holística del siste- ma agrícola del cáñamo}

\section{Zonificación de áreas de cultivos forestales para fines comerciales}

La identificación de zonas de cultivos forestales para fines comerciales se determinó por medio del estudio realizado por el Ministerio de Agricultura junto con entidades como la UPRA. Con el permiso académico generado por esta última, en la tabla 11 se contempla el número de hectáreas por región natural destinado a fines comerciales, de acuerdo con las condiciones geográficas (aptitud alta, media y baja) y las capacidades de producción de las plantaciones forestales con fines comerciales, en donde el termino de aptitud "hace referencia al grado de adecuación de una determinada unidad de tierra para un tipo específico de uso, calidad del aire rotación de cultivos, pastos limpios, entre otras características".

Tabla 11. Número de hectáreas (ha) con fines comerciales según regiones sectoriales y condiciones geográficas

\begin{tabular}{|c|c|c|c|}
\hline Región & Aptitud alta (total ha) & $\begin{array}{c}\text { Aptitud media } \\
\text { (total ha) }\end{array}$ & Aptitud baja (total ha) \\
\hline \multicolumn{2}{|c|}{ Andina } & 2.368 .809 & 2.168 .677 \\
\hline Caribe & 2.342 .724 & 1.229 .938 & 874.906 \\
\hline Pacífica & 684.416 & 810.226 & 680.136 \\
\hline Orinoquía & 597.912 & 1.369 .217 & 5.952 .642 \\
\hline Amazonía & 32.907 & 439.454 & 1.653 .354 \\
\hline Total ha & 7.258 .440 & 6.217 .643 & 11.329 .715 \\
\hline
\end{tabular}

Fuente: UPRA (2019). 
Aunque las condiciones climáticas para la siembra de ambos cultivos (pino y cáñamo) pueden llegar a ser las mismas, estos cuentan con rendimientos en su producción y condiciones agronómicas diferentes. Al realizar las comparaciones que se observan en la tabla 12 , el cáñamo se presenta como un material con potencial para la producción y comercialización a escala.

Tabla 12. Relaciones comparativas de los cultivos de pino y cáñamo

\begin{tabular}{|c|c|}
\hline Pino & Cáñamo \\
\hline 1.100 plantas/ha (ANDI) & 4 plantas/m², 40.000 plantas/ha (González-García et al., 2010b; \\
Mark \& Will, 2019)
\end{tabular}

Fuente: elaboración propia.

\section{Simulación sistema agrícola del cáñamo}

Los parámetros a tener en cuenta inicialmente varían en función de los tiempos de procesamiento, siembra y cosecha, así como de la capacidad máxima de procesamiento a una hectárea de cultivo. Del subsistema 1, "Producción agrícola", los parámetros iniciales considerados fueron: tiempo de germinación de semillas (3-4 semanas), tiempo de fertilización (2 semanas), tiempo de siembra (90-120 días) (Atmakuri et al., 2019) y tiempo de cosecha (2-4 días). Del subsistema 2, "Tratamiento de la fibra", los parámetros a contemplar son la eliminación de flores (o retted) y el secado (3 semanas), el embalado y el almacenamiento (formación de pacas de $325 \mathrm{~kg}$ ) (González-García et al., 2010b). La figura 14 presenta el modelo del sistema en el software FlexSim.

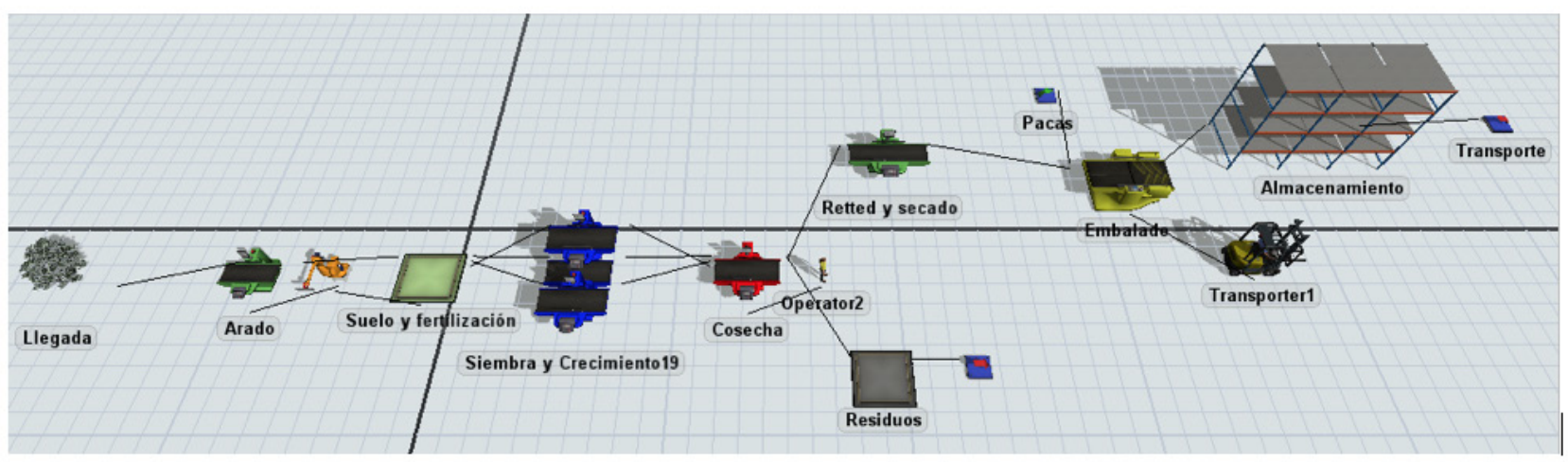

Figura 14. Modelo del sistema agrícola del cáñamo

Fuente: elaboración propia en el software FlexSim.

Al implementar el uso de operarios, ampliar la capacidad de las maquinarias de arado (permitiendo con ello disminuir el tiempo de trabajo), y el uso de maquinaria para la siembra y la fertilización como principales mejoras, se obtienen los análisis descritos en los siguientes apartados.

\section{Resumen y análisis de los resultados}

Solo se genera el $10 \%$ de los residuos del total del material destinado para el procesamiento de la fibra. En el almacenamiento, el $90 \%$ de la producción está lista para la distribución, como se observa en los resultados obtenidos por el simulador (figura 15). 


\section{Salidas}

WIP

Almacenamiento

Salidas Residuos

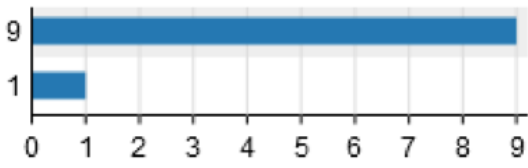

Figura 15. Porcentaje de eficiencia del sistema agrícola Fuente: elaboración propia en el software FlexSim.

Con el fin de procesar las toneladas requeridas de material por sectores - definidos a lo largo del trabajo como aptitudes alta, media y baja-, y contemplando que cada unidad procesada corresponde a una carga de $100 \mathrm{~kg}$ (para efectos de la simulación), para cumplir con los rendimientos de materia seca para el procesamiento de papel se requieren 200 unidades en la zona de aptitud alta, 140 en la zona de aptitud media y 100 en la de aptitud baja. En la tabla 13 se visualizan los resultados concernientes a las unidades producidas y los tiempos promedio de cada proceso.
Dado que los sistemas cumplen con el propósito inicial de cosechar todas las unidades (plantas por tonelada sembrada), las unidades que durante el proceso de secado son mayores se presentan en el sistema de aptitud baja, considerando que este procesó una mayor cantidad de material. Este sistema presentó una eficiencia de $93 \%$ en los procesos de cosecha y secado, en comparación con $87,9 \%$ del sistema de aptitud media y $89,5 \%$ del sistema de aptitud alta. Los tiempos promedios de todos los procesos permanecen estables en los tres sistemas, lo cual garantiza la eficiencia del sistema propuesto.

El hecho de incluir maquinaria para el desarrollo de los procesos en el sistema simulado permite determinar que los tiempos de ocio (tabla 14) no son relevantes. Sin embargo, el tiempo perdido en el trasporte o los movimientos realizados hasta la preparación de los procesos sí resultan ser altos.

Tabla 13. Capacidades y tiempos promedio en los procesos agrícolas de las plantaciones de cáñamo

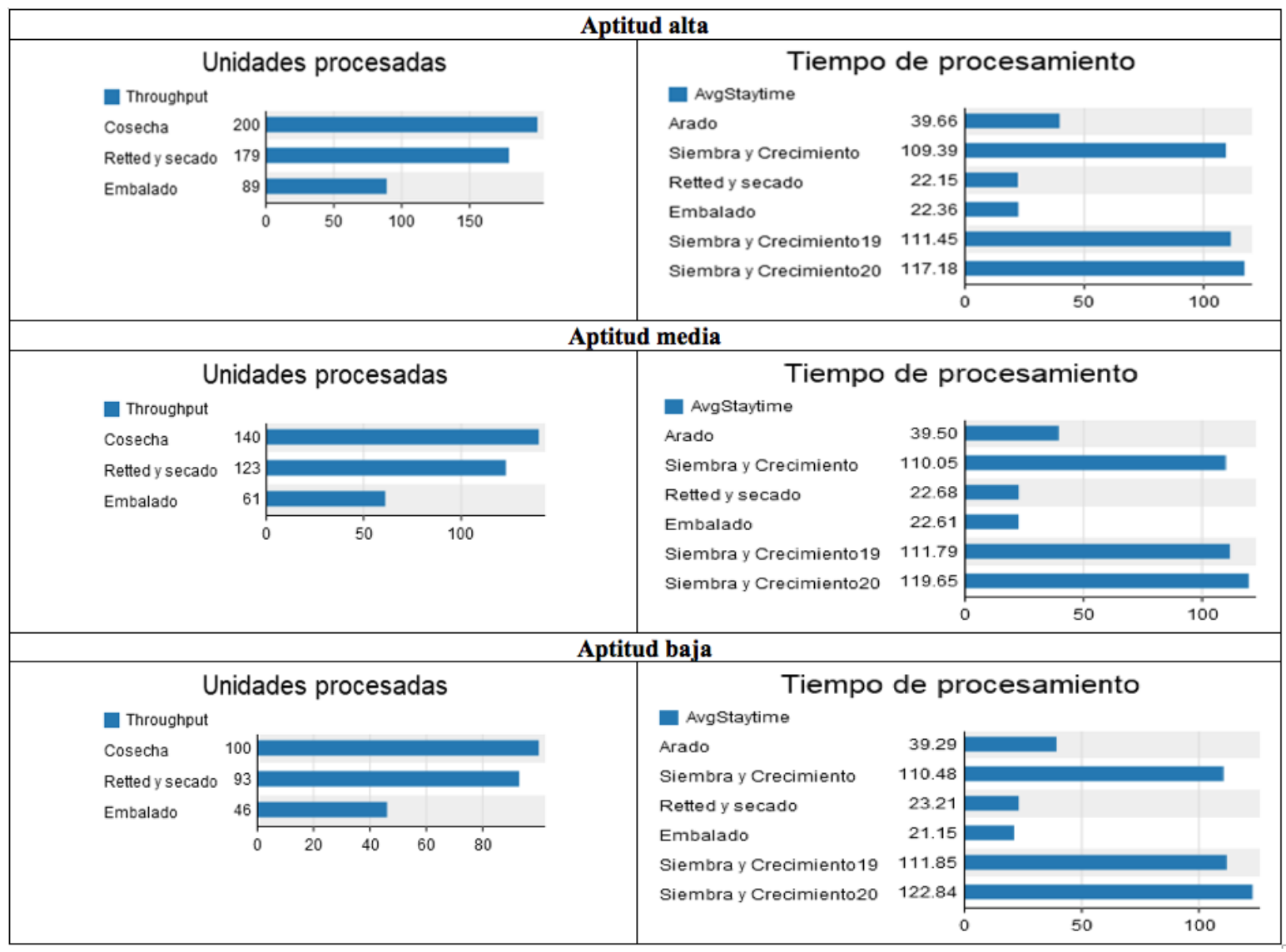

Fuente: elaboración propia en el software FlexSim. 
Tabla 14. Porcentajes de los tiempos de operación de las maquinarias empleadas en el sistema agrícola de cáñamo

\begin{tabular}{|c|c|c|}
\hline Aptitud alta & Aptitud media & Aptitud baja \\
\hline $\begin{array}{c}\text { State } \\
\text { Offset travel empty } \square \text { Offset travel loaded } \\
\text { Unloading Idle }\end{array}$ & $\begin{array}{c}\text { State } \\
\text { affset travel empty } \\
\text { anloading Offset travel loaded }\end{array}$ & $\begin{array}{c}\text { State } \\
\text { Offset travel empty } \square \text { Offset travel loaded } \\
\text { Unloading Idle }\end{array}$ \\
\hline $93.23 \%$ & $91.16 \%$ & $88.37 \%$ \\
\hline
\end{tabular}

Fuente: elaboración propia en el software FlexSim.

\section{Matriz multicriterio}

Para poder tomar una decisión con propiedad acerca de cuál es el sector regional que daría los mejores beneficios - no solo de tipo económico, sino también en términos de eficiencia de procesos, utilización y adquisición de recursos, posicionamiento estratégico y rendimientos, así como un futuro de inversión social一, se realizó la estimación de los criterios relevantes en la toma de decisiones empleando la metodología AHP. Así, se categorizó cada criterio de acuerdo con los grados de importancia de las opciones disponibles, como se observa en la tabla 15.

Tabla 15. Ponderación criterio-alternativa según el modelo AHP

\begin{tabular}{|c|c|c|c|c|c|c|c|}
\hline & \multicolumn{3}{|c|}{ Criterio: posicionamiento estratégico } & & \multicolumn{3}{|c|}{ Criterio: disposición de terreno } \\
\hline & Aptitud alta & Aptitud media & Aptitud baja & & Aptitud alta & Aptitud media & Aptitud baja \\
\hline Aptitud alta & & 2 & 6 & Aptitud alta & & 2 & \\
\hline Aptitud media & & & 3 & Aptitud media & & & \\
\hline \multirow[t]{3}{*}{ Aptitud baja } & & & & Aptitud baja & 4 & 5 & \\
\hline & \multicolumn{3}{|c|}{ Criterio: adquisición de recursos } & & \multicolumn{3}{|c|}{ Criterio: costos de producción } \\
\hline & Aptitud alta & Aptitud media & Aptitud baja & & Aptitud alta & Aptitud media & Aptitud baja \\
\hline Aptitud alta & & & 2 & Aptitud alta & & & 5 \\
\hline Aptitud media & 2 & & 2 & Aptitud media & 2 & & 6 \\
\hline \multirow[t]{3}{*}{ Aptitud baja } & & & & Aptitud baja & & & \\
\hline & \multicolumn{3}{|c|}{ Criterio: rendimientos de producción } & & \multicolumn{3}{|c|}{ Criterio: utilización de los recursos } \\
\hline & Aptitud Alta & Aptitud media & Aptitud baja & & Aptitud alta & Aptitud media & Aptitud baja \\
\hline Aptitud alta & & 3 & & Aptitud alta & & 2 & \begin{tabular}{|l|}
5 \\
\end{tabular} \\
\hline Aptitud media & & & & Aptitud media & & & 2 \\
\hline \multirow[t]{3}{*}{ Aptitud baja } & 7 & 8 & & Aptitud baja & & & \\
\hline & \multicolumn{3}{|c|}{ Criterio: eficiencia en los procesos } & & \multicolumn{3}{|c|}{ Criterio: tiempos de procesamiento } \\
\hline & Aptitud alta & Aptitud media & Aptitud baja & & Aptitud alta & Aptitud media & Aptitud baja \\
\hline Aptitud alta & & & & Aptitud alta & & 3 & 4 \\
\hline Aptitud media & 5 & & & Aptitud media & & & 3 \\
\hline Aptitud baja & 9 & 7 & & Aptitud baja & & & \\
\hline & & & \multicolumn{3}{|c|}{ Criterio: inversión social } & & \\
\hline & & & Aptitud alta & Aptitud media & Aptitud baja & & \\
\hline & & Aptitud alta & & & 1 & & \\
\hline & & Aptitud media & 5 & & 6 & & \\
\hline & & Aptitud baja & & & & & \\
\hline
\end{tabular}

Fuente: elaboración propia 


\section{Resumen y análisis de los resultados}

Para el desarrollo de la matriz se planteó como objetivo la "selección de la zona de inversión inicial", teniendo como criterios base los anteriormente expuestos y como alternativas las zonas de aptitud, alta, media y baja. Las ponderaciones entre los criterios se observan en la figura 16.

\begin{tabular}{|c|c|c|c|c|c|c|c|c|c|}
\hline & Posicionan & Disposición & Adquisiciór & Costos de F & Rendimien & Utilización & Eficiencia c & Tiempos de & Inversión s" \\
\hline Posicionamiento estratégico & & 1.0 & 3.0 & 2.0 & 3.0 & 1.0 & 2.0 & 3.0 & 4.0 \\
\hline Disposición de terreno & & & 2.0 & 2.0 & 3.0 & 3.0 & 2.0 & 1.0 & 4.0 \\
\hline Adquisición de recursos & & & & 1.0 & 1.0 & 1.0 & 2.0 & 3.0 & 6.0 \\
\hline Costos de producción & & & & & 2.0 & 1.0 & 2.0 & 3.0 & 5.0 \\
\hline Rendimientos de producción & & & & & & 2.0 & 3.0 & 3.0 & 3.0 \\
\hline Utilización de los recursos & & & & & & & 2.0 & 4.0 & 5.0 \\
\hline Eficiencia de los procesos & & & & & & & & 2.0 & 5.0 \\
\hline Tiempos de procesamiento & & & & & & & & & 5.0 \\
\hline Inversión social & Incon: $\mathbf{0 . 1 0}$ & & & & & & & & \\
\hline
\end{tabular}

Figura 16. Ponderación criterio-criterio según el modelo AHP.

Fuente: elaboración propia en el software Expert Choice.

El modelo se considera aceptable y con veracidad, ya que el índice de inconsistencia fue igual a 0,10 . Por consiguiente, se determina que el posicionamiento estraté- gico y el rendimiento en la producción (15,5 y $18.5 \%$, respectivamente) deben ser los criterios de selección de mayor importancia, como se muestra en la figura 17.
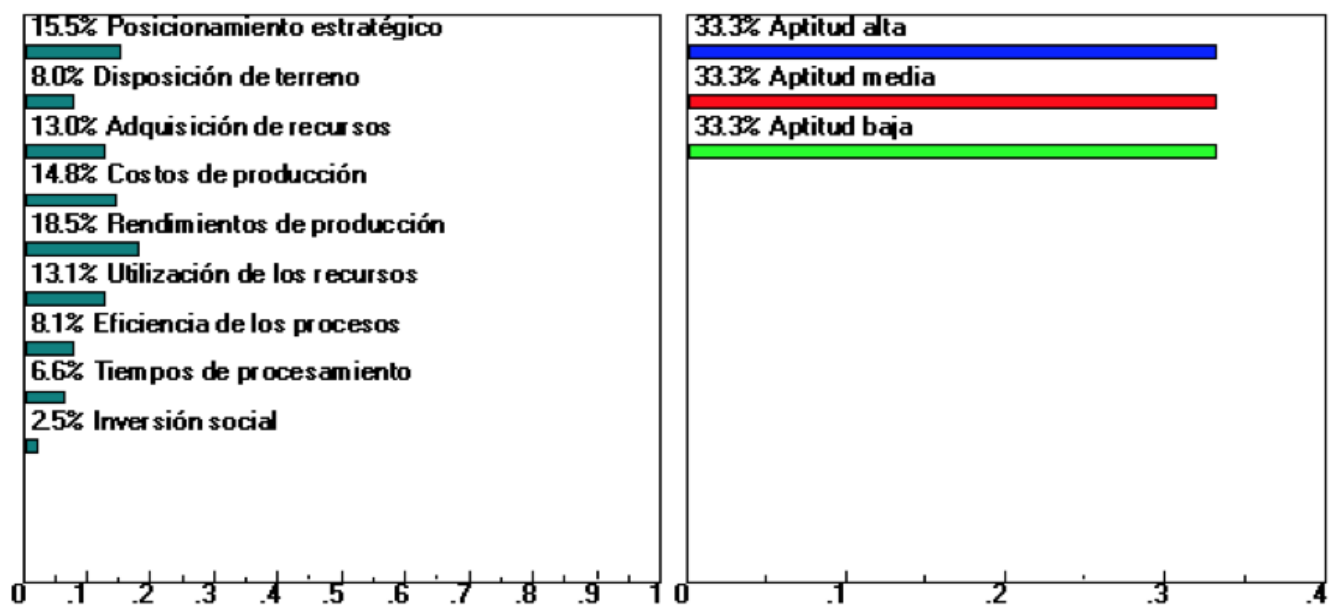

Figura 17. Análisis de sensibilidad dinámico Fuente: elaboración propia en el software Expert Choice.

Con base en los criterios de interés, se determina que la zona con aptitud alta es la mejor opción para el inicio del proyecto de inversión de cultivos forestales con fines comerciales, como se aprecia en la figura 18.

\section{CONCLUSIONES}

El cultivo de cáñamo se ha convertido en un modelo de desarrollo que abarca la sociedad, el ambiente y la economía, los principales ejes del desarrollo industrial. Se prevé que con el potencial de los cultivos de cáñamo se logrará apoyar a diversos sectores económicos, empezando desde los agricultores, emprendedores y empresarios, hasta llegar al consumidor final; en este caso, las partes integradas del sistema productivo del papel. Además, se ha demostrado 


\begin{tabular}{|l|l|l|l|}
\hline \multicolumn{1}{|c|}{ Criterio de selección 1: posicionamiento estratégico } & \multicolumn{2}{|c|}{ Criterio de selección 2: disposición del terreno } \\
\hline A ptitud alta & & \\
\hline Compare the relative importance with respect to: Posicionamiento
\end{tabular}

Figura 18. Comparación de los criterios de selección. Fuente: elaboración propia en el software Expert Choice.

que el cáñamo puede llegar a convertirse en un material potencial para todo tipo de industria (biorrefinería, alimentaria, textil, automotriz, entre muchas otras).

Se estima que en el futuro el cáñamo sustituirá parcial o totalmente las plantaciones forestales tradicionales (pino y eucalipto) destinadas para el comercio. En primera instancia, las propiedades fisicoquímicas del cáñamo representan un gran potencial productivo; sin mencionar que este se puede convertir en una solución a los problemas de deforestación nacional ocasionados por el incremento de la tala ilegal, empleada para el desarrollo de todo tipo de industrias.

Gracias a los proyectos ambientales y de reactivación económica que el Estado Colombiano ha venido fomentando, fue posible analizar el potencial del cáñamo como cultivo de producción en masa, demostrando que este cultivo cuenta con grandes perspectivas comerciales a escala nacional e internacional, debido al interés mundial por esta planta.

Gracias a la geografía de Colombia y a las condiciones climáticas, la calidad y economía de los procesos y la mano de obra del país - lo que a lo largo del trabajo se denominó zonas con aptitud alta-, la región Andina y gran parte de la región Caribe cuentan con más de 7 millones de hectáreas óptimas para la producción, comercialización y desarrollo de nuevos productos, lo que permitiría a esta industria innovar a futuro mediante el uso de biotecnologías verdes enfocadas en el desarrollo sostenible.

El estudio de zonificación de áreas forestales para fines comerciales realizado por la UPRA permitió establecer un punto de partida para la producción del cultivo de cáñamo como sustituto de materiales tradicionales de la industria papelera, lo cual representa un avance para futuros estudios financieros, productivos e industriales orientados hacia la innovación de la economía verde del país.

El proceso de obtención de papel de manera tradicional permite formular mejoras en los procesos de secado y laminado en aras de optimar las condiciones visuales del producto final. Así mismo, se recomienda perfeccionar el proceso de lavado, puesto que es posible disminuir el uso de químicos para mejorar la coloración del material, como se demostró en esta investigación, tras comprobar que al disminuir un lavado se obtiene material en su mayoría blanco, lo cual resulta favorable para la industria y el consumidor.

\section{REFERENCIAS}

Atmakuri, A., Palevicius, A., Griskevicius, P., \& Janušas, G. (2019). Investigation of mechanical properties of hemp and flax fibers hybrid composites for biomedical applications. Mechanika, 25(2), 149-155. https://doi.org/10.5755/j01. mech.25.2.22712

Becerra-Quiroz, A. P., Buitrago-Coca, A. L., \& Pinto-Baquero, P. (2016). Sostenibilidad del aprovechamiento del bagazo de caña de azúcar en el Valle del Cauca, Colombia. Ingeniería Solidaria, 12(20), 133-149. https://doi.org/10.16925/ in.v12i20.1548 
Cebreiros, F., Clavijo, L., Boix, E., Ferrari, M. D., \& Lareo, C. (2020). Integrated valorization of eucalyptus sawdust within a biorefinery approach by autohydrolysis and organosolv pretreatments. Renewable Energy, 149, 115-127. https://doi. org/10.1016/j.renene.2019.12.024

Chávez-Sifontes, M., \& Domine, M. E. (2010). Lignina, estructura y aplicaciones: Métodos de despolimerización para la obtención de derivados aromáticos de interés industrial. Avances en Ciencias e Ingeniería, 4(4),15-46. https://dialnet. unirioja.es/servlet/articulo?codigo $=4710101$

Dávila, J. A., Rosenberg, M., \& Cardona, C. A. (2017). A biorefinery for efficient processing and utilization of spent pulp of Colombian Andes Berry (Rubus glaucus Benth.): Experimental, techno-economic and environmental assessment. Bioresource Technology, 223, 227-236. https:// doi.org/10.1016/j.biortech.2016.10.050

Daza-Merchán, Z. T., Escudero-Agudelo, J., Gil-Zapata, N. J., \& Mora-Muñoz, O. Y. (2013). Evaluación de las enzimas celulolíticas producidas por hongosnativos mediante fermentación en estado sólido (SSF) utilizando residuos de cosecha de caña de azúcar. Revista Colombiana de Biotecnología, 15(1), 108-117. https://revistas.unal.edu.co/ index.php/biotecnologia/article/view/30844

Díaz-Martínez, M. A., \& Zárate-Cruz, R. (2018). Simulación Flexsim, una nueva alternativa para la ingeniería hacia la toma de decisiones en la operación de un sistema de múltiples estaciones de prueba. Revista Cientifica del Instituto Politécnico Nacional de México, 22(2), 1-17.

González-García, S., Hospido, A., Feijoo, G., \& Moreira, M. T. (2010a). Life cycle assessment of raw materials for non-wood pulp mills: Hemp and flax. Resources, Conservation and Recycling, 54(11), 923-930. https://doi.org/10.1016/j.resconrec.2010.01.011

González-García, S., Moreira, M. T., Artal, G., Maldonado, L., \& Feijoo, G. (2010b). Environmental impact assessment of non-wood based pulp production by soda-anthraquinone pulping process. Journal of Cleaner Production, 18(2), 137-145. https://doi. org/10.1016/j.jclepro.2009.10.008
González-Velandia, K. D., Daza-Rey, D., CaballeroAmado, P. A., \& Martínez-González, C. (2016). Evaluación de las propiedades físicas y químicas de residuos sólidos orgánicos a emplearse en la elaboración de papel. Luna Azul, 43(43), 499-517. https://doi.org/10.17151/luaz.2016.43.21

Han, J. S., \& Rowell, J. S. (2008). Chemical composition of fibers. Paper and Composites from Agro-Based Resources, 283, 83-134.

Jan, E. G. (2009). Environmental benefits of natural fibre production and use. Proceedings of the Symposium on Natural Fibres, 3-17. ftp://ftp.fao. org/docrep/fao/011/i0709e/i0709e03.pdf

Johnson, R. (2018). Hemp as an agricultural commodity. Congressional Research Service. https://fas.org/sgp/crs/misc/RL32725.pdf

Kitrytė, V., Bagdonaitè, D., \& Rimantas-Venskutonis, P. (2018). Biorefining of industrial hemp (Cannabis sativa L.) threshing residues into cannabinoid and antioxidant fractions by supercritical carbon dioxide, pressurized liquid and enzyme-assisted extractions. Food Chemistry, 267(March), 420-429. https://doi.org/10.1016/j.foodchem.2017.09.080

Komuraiah, A., Kumar, N. S., \& Prasad, B. D. (2014). Chemical composition of natural fibers and its influence on their mechanical properties. Mechanics of Composite Materials, 50(3), 359376. https://doi.org/10.1007/s11029-014-9422-2

Mark, T. B., \& Will, S. (2019). Economic issues and perspectives for industrial hemp. En D. W. Williams (ed.), Industrial hemp as a modern commodity crop (pp. 107-118). American Society of Agronomy, Crop Science Society of America, Soil Science Society of America https://doi.org/10.2134/industrialhemp. c7

Moonart, U., \& Utara, S. (2019). Effect of surface treatments and filler loading on the properties of hemp fiber/natural rubber composites. Cellulose, 26(12), 7271-7295. https://doi.org/10.1007/ s10570-019-02611-w

Organización Internacional de Normalización [Iso]. (2013). Papel y cartón. Determinación del gramaje (IsO 536:2012). IsO 
Plazonić, I., Barbarić-Mikočević, Ž., Bates, I., \& Malnar, L. (2016). Chemical stability of prints made on hemp fibre based papers. Acta Graphica: Journal for Printing Science and Graphic Communications, 27(3), 25-30.

Poisa, L., \& Adamovics, A. (2011). Evaluate of hemp (Cannabis sativa L.) quality parameters for bioenergy production. Engineering for Rural Development, 26, 358-362.

Sausserde, R., \& Adamovics, A. (2013). Industrial hemp for biomass production. Journal of Agricultural Engineering, 44(2s), 10-13. https:// doi.org/10.4081/jae.2013.s2.e123

Tavella, M. A., Miropolsky, A., \& Maner, R. (2016). Aplicación del software Expert Choice como herramienta de soporte de decisión para la localización sustentable de parques industriales. Tecnología y Ciencia, 28, 5-16.
Unidad de Planificación Rural Agropecuaria [UPRA]. (2018). Plantaciones forestales con fines comerciales para la obtención de madera y su cadena productiva. UPRA. https://bit.ly/36zrrd4

Unidad de Planificación Rural Agropecuaria [UPRA]. (2019). Zonificación para plantaciones forestales con fines comerciales. Escala 1:100.000. UPRA. https:// repository.agrosavia.co/handle/20.500.12324/34413

van-der-Werf, H. M. G., Harsveld-van-der-Veen, J. E., Bouma, A. T. M., \& Ten-Cate, M. (1994). Quality of hemp (Cannabis sativa L.) stems as a raw material for paper. Industrial Crops and Products, 2(3), 219-227. https://doi.org/10.1016/0926-6690(94)90039-6 